\title{
Raman tensor of layered black phosphorus
}

Yanming Zhu, Wei Zheng ${ }^{*}$ (D), Weiliang Wang, Siqi Zhu, Linxuan Li, Lu Cheng, Mingge Jin, Ying Ding and Feng Huang

\author{
* Correspondence: zhengw37@mail. \\ sysu.edu.cn \\ State Key Laboratory of \\ Optoelectronic Materials and \\ Technologies, School of Materials, \\ Sun Yat-sen University, Guangzhou \\ 510275, Guangdong, China
}

\begin{abstract}
Black phosphorus has a strong Raman anisotropy on the basal and cross planes due to its orthorhombic crystal structure. However, almost all the studies on black phosphorus' anisotropy focus on basal plane with the cross plane neglected. Here, we performed a systematic angle-resolved polarized Raman scattering on both the basal and cross planes of black phosphorus and obtained its integral Raman tensors. It is discovered that when the polarization direction of excitation light is along different crystal axes, the Raman intensity ratio $\left(I_{x x}: I_{y y}: I_{z z}\right)$ of $A_{g}^{1}$ mode is 256:1:5. Besides, via calculation, it is confirmed that the strong Raman anisotropy mainly comes from different differential polarizability alone different directions. This phenomenon is also observed when it comes to the $A_{g}^{2}$ mode.
\end{abstract}

Keywords: Black phosphorus, Polarized Raman spectrum, Differential polarizability, Raman tensor

\section{Introduction}

Recently, two-dimensional materials have attracted much attention [1, 2], especially its polarization characteristics [3-7], for which the high directional charge and energy transfer characteristics are important indicators to evaluate devices' performance. The directional selective characteristic of optoelectronic device originates from anisotropy of atomic arrangement. Based on symmetry theory, black phosphorus (BP) belongs to $D_{2 h}$ space group, which suggests that BP has different atomic arrangements along zigzag and armchair directions [8-11]. Therefore, BP becomes one of the excellent candidates to fabricate directional selective optoelectronic devices.

Raman polarization characteristic, as an important branch of anisotropy, is limited by corresponding Raman tensor which is a key index to evaluate Raman scattering intensity [12-14]. Thus, obtaining integral Raman tensor is significant to our understanding about physical properties of materials. Angle-resolved polarized Raman (APR) spectroscopy, as an undamaged tool, is powerful to measure Raman tensor. It has been performed in our previous work which studied the Raman polarization characteristic of anisotropic crystal, such as $\mathrm{MoS}_{2}$, black arsenic and AlN [2, 15-21]. Recently, BP has attracted much attention in the aspects of optical and electrical anisotropy. However, almost all the studies on the anisotropy of BP have put their focus on basal plane rather than cross plane so far [22-28].

(c) The Author(s). 2020 Open Access This article is licensed under a Creative Commons Attribution 4.0 International License, which permits use, sharing, adaptation, distribution and reproduction in any medium or format, as long as you give appropriate credit to the original author(s) and the source, provide a link to the Creative Commons licence, and indicate if changes were made. The images or other third party material in this article are included in the article's Creative Commons licence, unless indicated otherwise in a credit line to the material. If material is not included in the article's Creative Commons licence and your intended use is not permitted by statutory regulation or exceeds the permitted use, you will need to obtain permission directly from the copyright holder. To view a copy of this licence, visit http://creativecommons.org/licenses/by/4.0/. 
In this work, we systematically analyzed APR spectra of cross and basal planes of BP and discovered that $A_{g}^{1}$ and $A_{g}^{2}$ modes have strong Raman anisotropies. Raman intensity ratio of $A_{g}^{1}$ mode along different crystal axes $\left(I_{x x}: I_{y y}: I_{z z}\right)$ is up to $256: 1: 5$, and that of $A_{g}^{2}$ mode is 10:1:5. Via ab-initio calculation, we confirmed that it is the different differential polarizability along different crystal axes that determines anisotropic Raman intensity of $A_{g}$ mode.

\section{Experimental details}

Here, a backscattering geometry Renishaw spectrometer (inVia Reflx) was adopted to measure APR spectra of BP, where $488 \mathrm{~nm}$ laser was equipped as excitation light and focused on the basal or cross plane through $50 \times$ long focus quartz lens. Measured BP sample is a commercial single crystal with the size of $2 \mathrm{~mm}$, and the orientations of crystal axes are shown in Fig. 1a and b, where the basal and cross planes are corresponding to (010) and (100) planes respectively. During the measurement, the polarization directions of excitation light and scattering light were fixed and remained the same orientation, and layered BP sample was rotated in the step of $10^{\circ}$. Besides, to prevent local overheating from decomposition of sample, laser power density and acquisition time were set as $5 \mathrm{~mW}$ and $1 \mathrm{~s}$ respectively. In addition, integration number was set as 10 times to smooth experimental curves.

\section{Results and discussion}

Two representative Raman spectra corresponding to basal and cross planes are plotted in Fig. 1c and d. Collected APR spectra of the three modes $\left(A_{g}^{1}\left(363 \mathrm{~cm}^{-1}\right), A_{g}^{2}(470\right.$
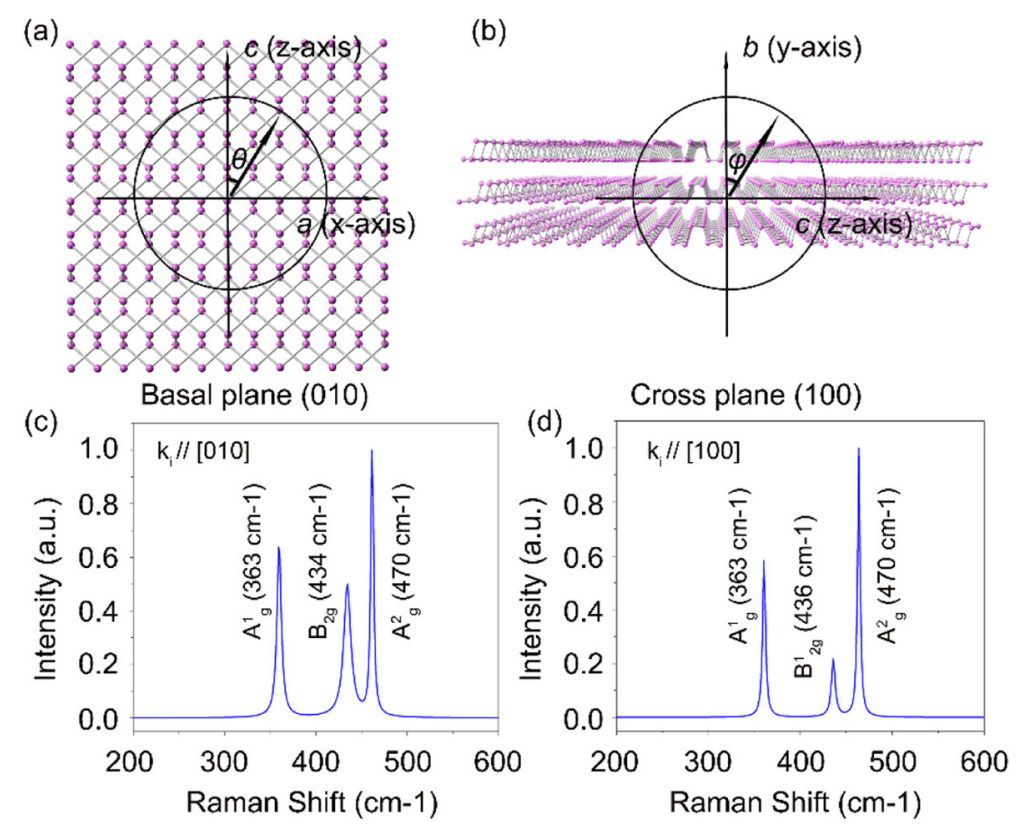

Fig. 1 Schematic of ARP Raman scattering of basal and cross planes of BP. a ARP Raman scattering of basal plane. $\theta$ is the angle between polarization direction of excitation light and $c$ axis. $\mathbf{b}$ APR scattering of cross plane. $\varphi$ is the angle between polarization direction of excitation light and $b$ axis. $\mathbf{c}$ and $\mathbf{d}$ denote representative Raman spectra of basal and cross planes 
$\left.\mathrm{cm}^{-1}\right)$ and $\left.B_{2 g}\left(434 \mathrm{~cm}^{-1}\right)\right)$ exhibit period changes when excitation light spreads along $b$-axis [010]. The situation is similar when excitation light spreads along $a$-axis [100]. Based on the definition of Raman intensity, $B_{2 g}$ mode will disappear in the Raman scattering process of cross plane, and $B_{3 g}^{1}$ mode cannot be observed in the Raman scattering process of basal plane. Therefore, $B_{2 g}$ and $B_{3 g}^{1}$ modes can be used as a criteria to distinguish crystal plane.

Based on the APR spectra of basal and cross planes, the Raman intensities of each mode at different rotation angles can be obtained. As shown in Fig. 2b, d, f and h, when the polarization orientation of excitation light locates on the basal plane, the Raman intensity ratio $\left(I_{x x}: I_{z z}\right)$ of $A_{g}^{1}$ mode is 11.1 , and that of $A_{g}^{2}$ mode is 1.94 . When the polarization orientation of excitation light locates on the cross plane, the Raman intensity ratio $\left(I_{z z}: I_{y y}\right)$ of $A_{g}^{1}$ mode is 23.1 and that of $A_{g}^{2}$ mode is 4.9. Combining the APR spectra of basal plane with those of cross plane, the Raman intensity ratios of $A_{g}^{1}$ and $A_{g}^{2}$ modes along different crystal axes can be derived, which are 256:1:23 and 9.6:1:4.9. It is obvious that there are strong Raman anisotropies of $A_{g}^{1}$ and $A_{g}^{2}$ modes, which can be elaborated with Raman selection rule and the definition of Raman intensity [22, 23].

Specifically, according to classical Raman scattering theory, Raman intensity $I$ for Raman active mode is given by [12].

$$
I \sim\left|\boldsymbol{e}_{s} \boldsymbol{R e}_{\boldsymbol{i}}\right|^{2}
$$

where $\boldsymbol{e}_{\boldsymbol{i}}$ and $\boldsymbol{e}_{s}$ denote polarization direction vectors of excitation and scattering light respectively. To explore the polarization characteristic of BP, $\boldsymbol{e}_{\boldsymbol{i}}$ is parallel to $\boldsymbol{e}_{s}$ throughout the whole measurement. $\boldsymbol{R}$ denotes Raman tensor, represented by a $3 \times 3$ matrix. According to group theory, the Raman tensors format of $A_{g}^{1}, A_{g}^{2}, B_{2 g}$ and $B_{3 g}^{1}$ modes can be written as $[12,23]$.
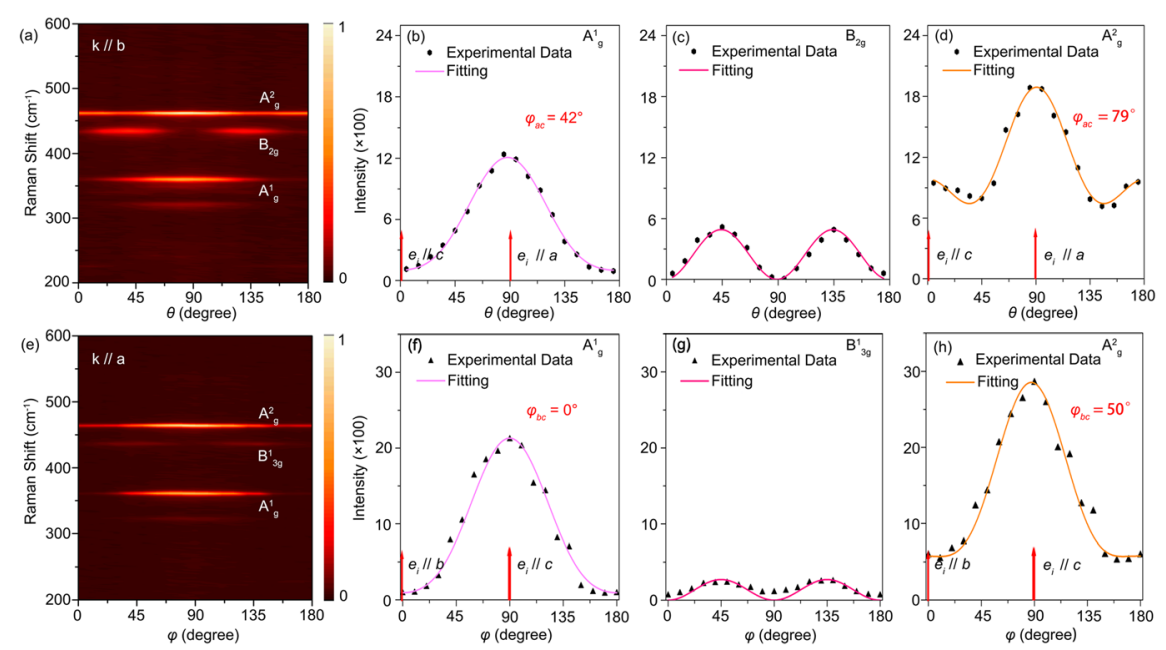

Fig. 2 ARP Raman scattering spectra of BP for (a)-(d) basal planes and (e)-(h) cross planes. ARP Raman scattering intensities are fitted with Eq. (4) and (6), and the phase differences between Raman tensor elements $a$ and $c$, that is the $\varphi_{a c}$ of $A_{g}^{1}$ and $A_{g}^{2}$ modes are $42^{\circ}$ and $79^{\circ}$ for basal plane. For cross plane, the phase differences between Raman tensor elements $b$ and $c$ of $A_{g}^{1}$ and $A_{g}^{2}$ modes are $0^{\circ}$ and $50^{\circ}$ respectively 

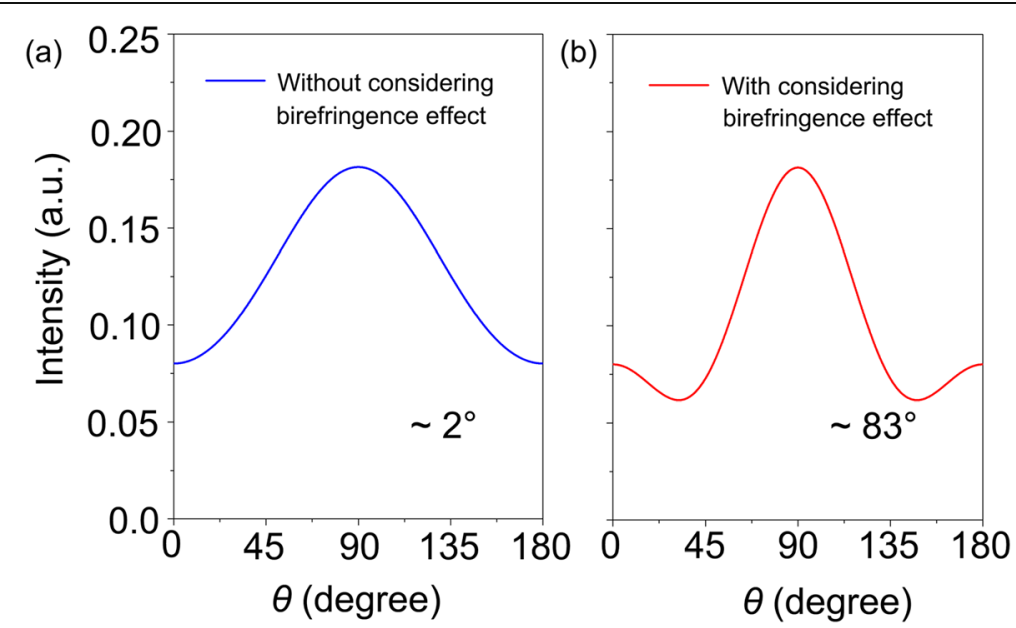

Fig. 3 Calculated basal plane APR spectra of $A_{g}^{2}$ mode with and without considering birefringence effect

$$
\begin{gathered}
R_{A_{g}^{1}}=\left(\begin{array}{ccc}
a_{1} e^{i \phi_{a_{1}}} & 0 & 0 \\
0 & b_{1} e^{i \phi_{b_{1}}} & 0 \\
0 & 0 & c_{1} e^{i \phi_{c_{1}}}
\end{array}\right), \\
R_{A_{g}^{2}}=\left(\begin{array}{ccc}
a_{2} e^{i \phi_{a_{2}}} & 0 & 0 \\
0 & b_{2} e^{i \phi_{b_{2}}} & 0 \\
0 & 0 & c_{2} e^{i \phi_{c_{2}}}
\end{array}\right) \\
R_{B_{2 g}}=\left(\begin{array}{lll}
0 & 0 & d \\
0 & 0 & 0 \\
d & 0 & 0
\end{array}\right) \\
R_{B_{3 g}^{1}}=\left(\begin{array}{lll}
0 & 0 & 0 \\
0 & 0 & f \\
0 & f & 0
\end{array}\right)
\end{gathered}
$$

where $a, b, c, d$ and $f$ represent the amplitudes of Raman tensor element, $\phi\left(\phi_{a_{1}}, \phi_{a_{2}}\right.$, $\phi_{b_{1}}, \phi_{b_{2}}, \phi_{c_{1}}$ and $\left.\phi_{c_{2}}\right)$ is the phase angle of Raman tensor element.

When the polarization direction of excitation and scattering light locates on the basal plane, $\boldsymbol{e}_{\boldsymbol{i}}$ and $\boldsymbol{e}_{s}$ can be written as.

$$
\boldsymbol{e}_{i}=\left(\begin{array}{c}
\sin \theta \\
0 \\
\cos \theta
\end{array}\right), \boldsymbol{e}_{s}=\left(\begin{array}{c}
\sin \theta \\
0 \\
\cos \theta
\end{array}\right),
$$

where $\theta$ denotes the rotation angle between the $a$-axis of BP and the polarization orientation of excitation (scattering) light. Based on Eq. (1), the function relations of Raman intensity of $A_{g}^{1}, A_{g}^{2}$ and $B_{2 g}$ modes with respect to rotation angle $\theta$ are given by

$$
\begin{gathered}
I_{A_{g}^{1}} \sim c_{1}^{2} \cos ^{4} \theta+a_{1}^{2} \sin ^{4} \theta+2 \mathrm{a}_{1} c_{1} \cos ^{2} \theta \sin ^{2} \theta \cos \phi_{a_{1} c_{1}} \\
I_{A_{g}^{2}} \sim c_{2}^{2} \cos ^{4} \theta+a_{2}^{2} \sin ^{4} \theta+2 a_{2} c_{2} \cos ^{2} \theta \sin ^{2} \theta \cos \phi_{a_{2} c_{2}} \\
I_{B_{2 g}} \sim d^{2} \sin ^{2} 2 \theta
\end{gathered}
$$

where $\phi_{a_{1} c_{1}}\left(=\phi_{a_{1}}-\phi_{c_{1}}\right)$ and $\phi_{a_{2} c_{2}}\left(=\phi_{a_{2}}-\phi_{c_{2}}\right)$ represent Raman phase differences between Raman tensor elements $a$ and $c$.

When the polarization direction vectors of excitation and scattering light locate on the cross plane, $\boldsymbol{e}_{\boldsymbol{i}}$ and $\boldsymbol{e}_{s}$ can be written as. 


$$
\boldsymbol{e}_{i}=\left(\begin{array}{c}
0 \\
\cos \phi \\
\sin \phi
\end{array}\right), \boldsymbol{e}_{s}=\left(\begin{array}{c}
0 \\
\cos \phi \\
\sin \phi
\end{array}\right),
$$

where $\phi$ denotes the rotation angle between the $b$ axis of BP and the polarization orientation of excitation light. Based on Eq. (1), the function relations of Raman intensity of $A_{g}^{1}, A_{g}^{2}$ and $B_{2 g}$ modes with respect to rotation angle $\phi$ are given by

$$
\begin{gathered}
I_{A_{g}^{1}} \sim b_{1}^{2} \cos ^{4} \phi+c_{1}^{2} \sin ^{4} \phi+2 b_{1} c_{1} \cos ^{2} \phi \sin ^{2} \phi \cos \phi_{b_{1} c_{1}}, \\
I_{A_{g}^{2}} \sim b_{2}^{2} \cos ^{4} \phi+c_{2}^{2} \sin ^{4} \phi+2 b_{2} c_{2} \cos ^{2} \phi \sin ^{2} \phi \cos \phi_{b_{2} c_{2}}, \\
I_{B_{3 g}^{1}} \sim f^{2} \sin ^{2} 2 \phi,
\end{gathered}
$$

where $\phi_{b_{1} c_{1}}\left(=\phi_{b_{1}}-\phi_{c_{1}}\right)$ and $\phi_{b_{2} c_{2}}\left(=\phi_{b_{2}}-\phi_{c_{2}}\right)$ represent Raman phase differences between Raman tensor elements $b$ and $c$.

According to the outline of the definition of Raman intensity, the Raman intensities of $A_{g}^{1}, A_{g}^{2}, B_{2 g}$ and $B_{3 g}^{1}$ modes satisfy the variation of Eq. (4) and Eq. (6) with the change of rotation angle. By fitting the APR spectra of $A_{g}^{1}, A_{g}^{2}, B_{2 g}$ and $B_{3 g}^{1}$ modes, the ratio relationship of Raman tensor elements can be observed, as shown in Table 1. For $A_{g}^{1}$ mode, the ratio of Raman tensor elements amplitudes $a_{1}$ to $c_{1}$ is 3.33 , and that of $c_{1}$ to $b_{1}$ is 4.8. Consequently, Raman tensor elements amplitudes $a_{1}, b_{1}$ and $c_{1}$ satisfy the relationship of $a_{1}>c_{1}>b_{1}$. Similarly, for $A_{g}^{2}$ mode, its diagonal Raman tensor elements amplitudes also satisfy the relationship of $a_{2}>c_{2}>b_{2}$.

Raman tensor element $R_{i j}^{q}$ is also defined as the derivative of susceptibility $\chi_{i j}$ with regard to atom position [12], thus

$$
R_{i j}^{q}=V_{\text {prim }} \sum_{\mu=1}^{N} \sum_{l=1}^{3} \frac{\partial X_{i j}}{\partial r_{l}(\mu)} \frac{e_{l}^{q}(\mu)}{\sqrt{M_{\mu}}},
$$

where $M_{\mu}$ and $V_{\text {prim }}$ represent the atomic mass of $\mu$ th atom and volume of unit cell respectively; $r_{l}(\mu)$ is the position of $\mu$ th atom along $l$ direction and $e_{l}^{q}(\mu)$ is the eigenvector of $q$ th phonon of $\mu$ th atom. Since polarizability $\alpha_{i j}$ is linear with susceptibility $\chi_{i j}$ $\left(\alpha_{i j}=\varepsilon_{0} X_{i j}\right)$, Raman tensor element $R_{i j}^{q}$ can be written as

$$
R_{i j}^{q}=V_{\text {prim }} \sum_{\mu=1}^{N} \sum_{l=1}^{3} \frac{\partial \alpha_{i j}}{\partial r_{l}(\mu)} \frac{e_{l}^{q}(\mu)}{\varepsilon_{0} \sqrt{M_{\mu}}} .
$$

where $\partial \alpha_{i j} / \partial r_{l}(\mu)$ can be defined as the differential polarizability with Raman intensity directly reflected. Take $A_{g}^{1}$ mode as an example, the differential polarizability along $a$ axis is larger than that along $c$-axis, which determines that there will be a larger Raman intensity when the polarization direction of excitation light is parallel to $a$-axis.

In addition, the susceptibility $\chi_{i j}$ and relative permittivity $\varepsilon_{i j}$ are interrelated $\left(\chi_{i j}=\varepsilon_{i j}\right.$ 1). Thus, Raman tensor element can also be written as

$$
R_{i j}^{q}=V_{p r i m} \sum_{\mu=1}^{N} \sum_{l=1}^{3} \frac{\partial \varepsilon_{i j}}{\partial r_{l}(\mu)} \frac{e_{l}^{q}(\mu)}{\sqrt{M_{\mu}}} .
$$

More importantly, the relative permittivity $\varepsilon_{i j}$ can be calculated by ab-inito calculation. The Raman tensor elements of $A_{g}^{1}$ and $A_{g}^{2}$ modes can be calculated by the vari- 
Table 1 Raman tensors obtained via APR scattering spectra and first-principle calculation

\begin{tabular}{|c|c|c|c|c|}
\hline \multirow{2}{*}{$\frac{\text { Mode }}{A_{g}^{1}}$} & \multirow{2}{*}{$\begin{array}{l}\text { Experimental } \\
/\end{array}$} & \multicolumn{3}{|l|}{ Calculated } \\
\hline & & $\left(\begin{array}{c}0.064 e^{i 0.24 \pi} \\
0 \\
0\end{array}\right.$ & $\begin{array}{c}0 \\
0.025 e^{i 0.05 \pi} \\
0\end{array}$ & $\begin{array}{c}0 \\
0 \\
0.033 \cdot e^{i 0.01 \pi}\end{array}$ \\
\hline & $a_{1} / c_{1}=3.33$ & \multicolumn{3}{|l|}{$a_{1} / c_{1}=1.94$} \\
\hline & $b_{1} / c_{1}=0.21$ & \multicolumn{3}{|l|}{$b_{1} / c_{1}=0.76$} \\
\hline & $\varphi_{a_{1} c_{1}}=0.23 \pi$ & \multicolumn{3}{|l|}{$\varphi_{a_{1} c_{1}}=0.23 \pi$} \\
\hline & $\varphi_{b_{1} c_{1}}=0$ & \multicolumn{3}{|c|}{$\varphi_{b_{1} c_{1}}=0.03 \pi$} \\
\hline \multirow[t]{2}{*}{$B_{2 g}$} & / & $\left(\begin{array}{cc}0 & 0 \\
0 & 0 \\
0.08 & 0\end{array}\right.$ & $\left.\begin{array}{c}0.08 \\
0 \\
0\end{array}\right)$ & \\
\hline & $d$ & \multicolumn{3}{|l|}{$d=0.08$} \\
\hline \multirow[t]{2}{*}{$B_{3 g}^{1}$} & / & $\left(\begin{array}{cc}0 & 0 \\
0 & 0 \\
0 & 0.03\end{array}\right.$ & $\left.\begin{array}{c}0 \\
0.03 \\
0\end{array}\right)$ & \\
\hline & $f$ & \multicolumn{3}{|l|}{$f=0.03$} \\
\hline \multirow[t]{5}{*}{$A_{g}^{2}$} & / & $\left(\begin{array}{c}0.43 e^{j 0.29 \pi} \\
0 \\
0\end{array}\right.$ & $\begin{array}{c}0 \\
0.0045 e^{i 0.04 \pi} \\
0\end{array}$ & $\left.\begin{array}{c}0 \\
0 \\
0.28 e^{j 0.3 \pi}\end{array}\right)$ \\
\hline & $a_{2} / c_{2}=1.39$ & \multicolumn{3}{|l|}{$a_{2} / c_{2}=1.54$} \\
\hline & $b_{2} / c_{2}=0.45$ & \multicolumn{3}{|l|}{$b_{2} / c_{2}=0.016$} \\
\hline & $\varphi_{a_{2} c_{2}}=0.44 \pi$ & \multicolumn{3}{|l|}{$\varphi_{a_{2} c_{2}}=0.01 \pi$} \\
\hline & $\varphi_{b_{2} c_{2}}=0.28 \pi$ & \multicolumn{3}{|l|}{$\varphi_{b_{2} c_{2}}=0.26 \pi$} \\
\hline
\end{tabular}

ation of relative permittivity before and after corresponding vibration. Here, the Raman tensor elements of various vibration modes are calculated via Vienna Ab-initio Simulation Package (VASP) [29-40] and phonon calculation software PHONOPY. During the density functional perturbation theory (DFPT) calculation, a $3 \times 3 \times 3$ supercell was adopted to obtain the force constants. A projector augmented wave (PAW) basis set with $500 \mathrm{eV}$ cutoff was adopted, and Perdew-Burke-Ernzerhof functional was used as pseudopotentials. The relaxation of electron cannot be stopped until the free energy change is smaller than $10^{-8} \mathrm{eV}$ and the force between two steps is smaller than $10^{-3}$ $e V / \AA$. A high-density $k$-mesh of $50 \times 1 \times 50$ was performed for self-consistent calculation to obtain inductance coefficient tensors, as shown in Table 1. For $A_{g}^{1}$ mode, the calculated Raman tensor elements amplitudes ratio of $a_{1}$ to $c_{1}$ is 1.94 , and that of $c_{1}$ to $b_{1}$ is 1.32 . Therefore, $a_{1}, b_{1}$ and $c_{1}$ satisfy the relationship of $a_{1}>c_{1}>b_{1}$, which is in accordance with measured result. Similarly, the relationship among Raman tensor elements amplitudes $a_{2}, b_{2}$ and $c_{2}$ also satisfy $a_{2}>c_{2}>b_{2}$. Based on ab-initio calculation, it can be concluded that the strong Raman anisotropies of $A_{g}^{1}$ and $A_{g}^{2}$ modes come from different differential polarizabilities along different crystal axes.

Besides, the phase angle of Raman tensor element can also be calculated via ab-initio calculation. As we have claimed above, Raman tensor element is proportional to the derivative of the relative permittivity $\varepsilon_{i j}$ with respect to spatial position of atoms. However, relative permittivity $\varepsilon_{i j}$ is usually composed of a real part $\varepsilon_{i j}^{\prime}$ and an imaginary part $\varepsilon_{i j}^{\prime}$, leading to a correction of the expression of Raman tensor element, that is 


$$
R_{i j}^{q}=V_{p r i m} \sum_{\mu=1}^{N} \sum_{l=1}^{3} \frac{\partial\left(\varepsilon_{i j}^{\prime}+i \varepsilon_{i j}^{\prime \prime}\right)}{\partial r_{l}(\mu)} \frac{e_{l}^{q}(\mu)}{\sqrt{M_{\mu}}} .
$$

Calculated results are shown in Table 1 , where the Raman phase differences $\left(\phi_{a_{1} c_{1}}\right.$, $\phi_{b_{1} c_{1}}, \phi_{a_{2} c_{2}}, \phi_{b_{2} c_{2}}$ ) of $A_{g}^{1}$ and $A_{g}^{2}$ are $0.23 \pi, 0.03 \pi, 0.01 \pi, 0.26 \pi$ respectively.

By fitting the APR spectra of $A_{g}^{1}$ and $A_{g}^{2}$ modes based on Eq. (4) and Eq. (6), the phase angels can also be derived. The Raman phase differences $\phi_{a_{1} c_{1}}$ and $\phi_{b_{1} c_{1}}$ of $A_{g}^{1}$ modes are $0.23 \pi$ and 0 , and those of $A_{g}^{2}$ mode are $0.44 \pi$ and $0.28 \pi$. Compared with the calculated $\phi_{a_{2} c_{2}}$, the experimental $\phi_{a_{2} c_{2}}$ is much larger, which has puzzled us for a long time. In the Raman scattering process, except for Raman tensor, test environment and the property of materials will also affect the Raman scattering intensity. It seems difficult to find an acceptable reason to explain the large difference between experimental and theoretical values of $\phi_{a_{2} c_{2}}$.

Recently, a birefringence theory $[41,42]$ has been proposed to elaborate the Raman phase difference in transparent crystal, which has been confirmed by the materials such as AlN, GaN $[16,43,44]$. It is generally believed that due to a nearly negligible penetration depth, the theory is invalid in narrow band-gap materials. However, this understanding may be incomplete. For opaque optical crystals, the birefringence effect may also have a significant modulation influence on Raman phase difference. According to the definition of Raman scattering intensity and the deduction in Supporting Information, a phase factor $\phi$ and a constant $\boldsymbol{w}$ could be introduced into the Raman scattering intensity expression of $A_{g}^{2}$ mode in Eq. (4). Thus, when excitation light propagates along $b$-axis, Raman scattering intensity of $A_{g}^{2}$ mode can be written as

$$
I_{A_{g}^{2}} \sim c_{2}^{2} \cos ^{4} \phi+a_{2}^{2} \sin ^{4} \phi+2 a_{2} c_{2} \cdot \boldsymbol{w} \cdot \cos ^{2} \phi \sin ^{2} \phi \cos \left(\phi_{a_{2}}-\phi_{c_{2}}-\phi\right),
$$

where $\phi$ and $\boldsymbol{w}$ are functions respect to refractivity and extinction coefficient, given by

$$
\begin{array}{r}
\phi=\operatorname{arctg}\left(\frac{n_{x r} n_{z i}-n_{x i} n_{z r}}{n_{x i} n_{z r}+n_{x r} n_{z i}}\right), \\
\boldsymbol{w}=\frac{\frac{n_{x r} n_{z i}-n_{x i} n_{z r}}{\left(n_{x i}^{2}+n_{x r}^{2}\right)\left(n_{z i}^{2}+n_{z r}^{2}\right)}}{\sin \phi},
\end{array}
$$

where $n_{x r}$ and $n_{z r}$ denote refractive index along $x$ and $z$ directions respectively, and $n_{x i}$ and $n_{z i}$ are extinction coefficients along $x$ and $z$ directions respectively. As shown in Fig. 3, considering the birefringence effect, the theoretical value of Raman phase difference $\phi_{a_{2} c_{2}}$ of $A_{g}^{2}$ mode is $83^{\circ}$, which is close to experimental Raman phase difference $79^{\circ}$. Although BP is an opaque material relative to $488 \mathrm{~nm}$ and its penetration depth is very small, the effect of birefringence on the modulation of Raman phase difference cannot be ignored.

\section{Conclusions}

In this work, we analyzed the APR spectra of basal and cross planes of BP systematically with complete Raman tensors obtained. In addition, the strong Raman anisotropies of $A_{g}^{1}$ and $A_{g}^{2}$ modes were discovered in this research. Via first-principle calculation, the 
strong anisotropy has been confirmed as originating from the different differential polarizability along different crystal axes. Besides, it is found that when excitation light propagates along $b$-axis, the calculated Raman phase difference of $A_{g}^{2}$ mode is different from the experimental value, which may be owing to the modulation of birefringence effect.

\section{Supplementary information}

Supplementary information accompanies this paper at https://doi.org/10.1186/s43074-020-00017-7.

Additional file 1.

Acknowledgements

We thank the language editing from Miss Yang.

\section{Authors' contributions}

Yanming Zhu designed the experimental details and performed calculations. Siqi Zhu, Linxuan Li, Lu Cheng, Mingge Jin, Ying Ding performed the experiments. Weiliang Wang, Wei Zheng and Feng Huang directed the program. The author(s) read and approved the final manuscript.

\section{Funding}

The financial support of this work is from the National Natural Science Foundation of China (Nos. 91333207, 61427901, 61604178, 91833301 and U1505252).

\section{Availability of data and materials}

The datasets used and analyzed during the current study are available from the corresponding author on reasonable request.

\section{Competing interests}

All financial and non-financial competing interests must be declared in this section.

Received: 2 April 2020 Accepted: 6 July 2020

Published online: 20 July 2020

\section{References}

1. Zheng W, Li F, Li G, Liang Y, Ji X, Yang F, Zhang Z, Huang F. Laser Tuning in van der Waals Crystals. ACS Nano. 2018;12: $2001-7$.

2. Zheng W, Yan J, Li F, Huang F. Elucidation of "phase difference" in Raman tensor formalism. Photon Res. 2018;6:709-12.

3. Chen Y, Chen C, Kealhofer R, Liu H, Yuan Z, Jiang L, Suh J, Park J, Ko C, Choe HS, Avila J, Zhong M, Wei Z, Li J, Li S, Gao H, Liu Y, Analytis J, Xia Q, Asensio MC, Wu J. Black arsenic: a layered semiconductor with extreme in-plane anisotropy. Adv Mater. 2018;30:1800754.

4. Chenet DA, Aslan OB, Huang PY, Fan C, van der Zande AM, Heinz TF, Hone JC. In-plane anisotropy in mono- and fewlayer ReS $S_{2}$ probed by Raman spectroscopy and scanning transmission Electron microscopy. Nano Lett. 2015;15:5667-72.

5. Yao Y, Zhang Y, Xiong W, Wang Z, Sendeku MG, Li N, Wang J, Huang W, Wang F, Zhan X, Yuan S, Jiang C, Xia C, He J. Growth and Raman scattering investigation of a new 2D MOX material: YbOCl. Adv Funct Mater. 2019;29:1903017.

6. Zhong X, Lee K, Meggiolaro D, Dismukes AH, Choi B, Wang F, Nuckolls C, Paley DW, Batail P, De Angelis F, Roy X, Zhu $X-Y . M_{6} S_{3} B r_{6}$ : an anisotropic 2D Superatomic semiconductor. Adv Funct Mater. 2019;29:1902951.

7. Huang S, Tatsumi Y, Ling X, Guo H, Wang Z, Watson G, Puretzky AA, Geohegan DB, Kong J, Li J, Yang T, Saito R, Dresselhaus MS. In-plane optical anisotropy of layered gallium telluride. ACS Nano. 2016;10:8964-72.

8. Khandelwal A, Mani K, Karigerasi MH, Lahiri I. Phosphorene - the two-dimensional black phosphorous: properties, synthesis and applications. Mater Sci Eng B. 2017;221:17-34.

9. Gupta A, Sakthivel T, Seal S. Recent development in 2D materials beyond graphene. Prog Mater Sci. 2015;73:44-126.

10. Li L, Yu Y, Ye GJ, Ge Q, Ou X, Wu H, Feng D, Chen XH, Zhang Y. Black phosphorus field-effect transistors. Nat Nanotechnol. 2014;9:372.

11. Tran V, Soklaski R, Liang Y, Yang L. Layer-controlled band gap and anisotropic excitons in few-layer black phosphorus. Phys Rev B. 2014:89:235319.

12. Cardona M. Resonance phenomena. In: Light Scattering in Solids II: Basic Concepts and Instrumentation (eds Cardona M, Güntherodt G). Springer Berlin Heidelberg (1982).

13. Loudon R. The Raman effect in crystals. Adv Phys. 2001;50:813-64

14. Raman CV. A new radiation. Indian J Phys. 1928;2:387-98.

15. Zheng W, Zheng RS, Wu HL, Li FD. Strongly anisotropic behavior of $A_{1}(T O)$ phonon mode in bulk AlN. J Alloy Compd. 2014:584:374-6.

16. Zheng W, Zheng R, Huang F, Wu H, Li F. Raman tensor of AlN bulk single crystal. Photon Res. 2015;3:38-43.

17. Zheng W, Zhu Y, Li F, Huang F. Raman spectroscopy regulation in van der Waals crystals. Photon Res. 2018;6:991-5.

18. Ding Y, Zheng W, Lin Z, Zhu R, Jin M, Zhu Y, Huang F. Raman tensor of layered WS 2 . Sci China Mater. 2020. https://doi. org/10.1007/s40843-020-1321-4.

19. Ding Y, Zheng W, Jin M, Zhu Y, Zhu R, Lin Z, Huang F. Raman tensor of layered MoS 2. Opt Lett. 2020;45:1313-6. 
20. Jin M, Zheng W, Ding Y, Zhu Y, Wang W, Huang F. Raman Tensor of van der Waals MoSe $e_{2}$.J Phys Chem Lett. 2020;11: $4311-6$.

21. Jin $M$, Zheng W, Ding $Y$, Zhu Y, Wang W, Huang F. Raman tensor of $W_{S} e_{2}$ via angle-resolved polarized Raman spectroscopy. J Phys Chem C. 2019;123:29337-42.

22. Ribeiro HB, Pimenta MA, de Matos CJS, Moreira RL, Rodin AS, Zapata JD, de Souza EAT, Castro Neto AH. Unusual angular dependence of the Raman response in black phosphorus. ACS Nano. 2015;9:4270-6.

23. Ribeiro HB, Pimenta MA, de Matos CJS. Raman spectroscopy in black phosphorus. J Raman Spectrosc. 2018;49:76-90.

24. Wang $T$, Liu J, Xu B, Wang R, Yuan $P$, Han M, Xu S, Xie Y, Wu Y, Wang X. Identifying the crystalline orientation of black phosphorus by using Optothermal Raman spectroscopy. ChemPhysChem. 2017;18:2828-34.

25. Wu J, Mao N, Xie L, Xu H, Zhang J. Identifying the crystalline orientation of black phosphorus using angle-resolved polarized Raman spectroscopy. Angew Chem. 2015;127:2396-9.

26. Ling X, Huang S, Hasdeo EH, Liang L, Parkin WM, Tatsumi Y, Nugraha ART, Puretzky AA, Das PM, Sumpter BG, Geohegan DB, Kong J, Saito R, Drndic M, Meunier V, Dresselhaus MS. Anisotropic Electron-photon and Electron-phonon interactions in black phosphorus. Nano Lett. 2016;16:2260-7.

27. Mao N, Wu J, Han B, Lin J, Tong L, Zhang J. Birefringence-directed Raman selection rules in 2D black phosphorus crystals. Small. 2016;12:2627-33.

28. Ribeiro HB, Villegas CEP, Bahamon DA, Muraca D, Castro Neto AH, de Souza EAT, Rocha AR, Pimenta MA, de Matos CJS. Edge phonons in black phosphorus. Nat Commun. 2016;7:12191.

29. Tóbik J, Tosatti E. Raman tensor calculation for magnesium phthalocyanine. Surf Sci. 2006;600:3995-8.

30. Zvereva EE, Shagidullin AR, Katsyuba SA. Ab initio and DFT predictions of infrared intensities and Raman activities. J Phys Chem A. 2011;115:63-9.

31. Saboori S, Deng Z, Li Z, Wang W, She J. $\beta$-As monolayer: vibrational properties and Raman spectra. ACS Omega. 2019;4: $10171-5$.

32. Irmer G, Röder C, Himcinschi C, Kortus J. Raman tensor elements and Faust-Henry coefficients of wurtzite-type a-GaN: how to overcome the dilemma of the sign of Faust-Henry coefficients in a-GaN? J Appl Phys. 2014;116:245702.

33. Blöchl P. Projector Agmented-Wave Method Phys Rev B 1994:50:17953-17979.

34. Hohenberg P, Kohn W. Inhomogeneous Electron gas. Phys Rev. 1964;136:B864.

35. Kresse G, Joubert D. From Ultrasoft Pseudopotentials to the Projector Augmented-Wave Method. Phys Rev B. 1999;59: 1758.

36. Kohn W, Sham $L$. Self-consistent equations including exchange and correlation effects. Phys Rev. 1965;140:A1133-8.

37. Kresse G, Furthmuller J. Efficient iterative schemes for ab initio total-energy calculations using a plane-wave basis set. Phys Rev B. 1996;54:11169-86.

38. Kresse G, Furthmüller J. Efficiency of ab-initio total energy calculations for metals and semiconductors using a planewave basis set. Comput Mater Sci. 1996;6:15-50.

39. Monkhorst HJ, Pack JD. On special points for Brillouin zone integrations. Phys Rev B. 1976;13:5188-92.

40. Perdew JP, Burke K, Ernzerhof M. Generalized Gradient Approximation Made Simple. Phys Rev Lett. 1996;77:3865-8.

41. Kranert C, Sturm C, Schmidt-Grund R, Grundmann M. Raman tensor formalism for optically anisotropic crystals. Phys Rev Lett. 2016;116:127401.

42. Kranert C, Sturm C, Schmidt-Grund R, Grundmann M. Raman tensor elements of $\beta$-Ga2O3. Sci Rep. 2016;6:35964

43. Sander T, Eisermann S, Meyer BK, Klar PJ. Raman tensor elements of wurtzite ZnO. Phys Rev B. 2012;85:165208.

44. Strach T, Brunen J, Lederle B, Zegenhagen J, Cardona M. Determination of the phase difference between the Raman tensor elements of the $\mathrm{A}_{19}$-like phonons in $\mathrm{SmBa}_{2} \mathrm{Cu}_{3} \mathrm{O}_{7-\delta}$. Phys Rev B. 1998;57:1292-7.

\section{Publisher's Note}

Springer Nature remains neutral with regard to jurisdictional claims in published maps and institutional affiliations.

\section{Submit your manuscript to a SpringerOpen ${ }^{\circ}$ journal and benefit from:}

- Convenient online submission

- Rigorous peer review

- Open access: articles freely available online

- High visibility within the field

- Retaining the copyright to your article

Submit your next manuscript at $\boldsymbol{\nabla}$ springeropen.com 